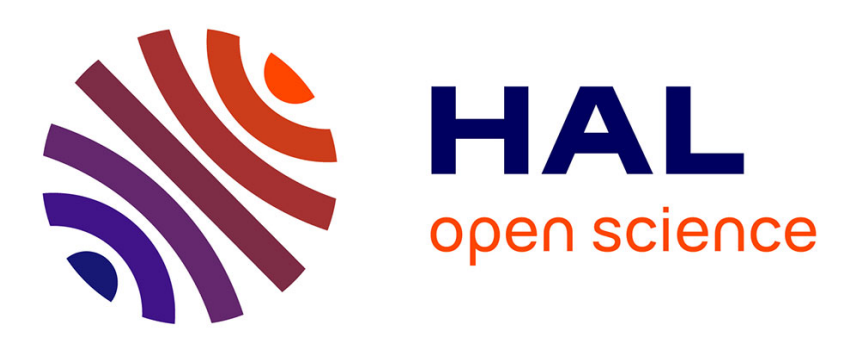

\title{
Magnetism and Morphology in Faceted B2-ordered FeRh Nanoparticles
}

\author{
Miao Liu, Patrizio Benzo, Hao Tang, Marion Castiella, Bénédicte \\ Warot-Fonrose, Nathalie Tarrat, Christophe Gatel, Marc Respaud, Joseph \\ Morillo, Marie-José Casanove
}

\section{To cite this version:}

Miao Liu, Patrizio Benzo, Hao Tang, Marion Castiella, Bénédicte Warot-Fonrose, et al.. Magnetism and Morphology in Faceted B2-ordered FeRh Nanoparticles. EPL - Europhysics Letters, 2016, 116 (2), pp.27006. hal-01426616

\section{HAL Id: hal-01426616 https://hal.science/hal-01426616}

Submitted on 4 Jan 2017

HAL is a multi-disciplinary open access archive for the deposit and dissemination of scientific research documents, whether they are published or not. The documents may come from teaching and research institutions in France or abroad, or from public or private research centers.
L'archive ouverte pluridisciplinaire HAL, est destinée au dépôt et à la diffusion de documents scientifiques de niveau recherche, publiés ou non, émanant des établissements d'enseignement et de recherche français ou étrangers, des laboratoires publics ou privés. 


\title{
Magnetism and Morphology in Faceted B2-ordered FeRh Nanoparticles
}

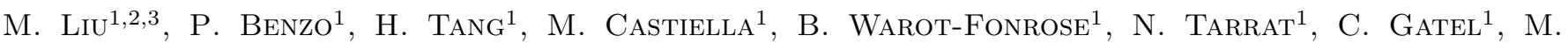 \\ Respaud $^{4}$, J. Morillo ${ }^{1}$ and M.J. CAsAnove ${ }^{1}$ (a) \\ 1 CEMES, CNRS UPR 8011 and Université de Toulouse, 29 rue Jeanne Marvig, F-31055 Toulouse, France \\ 2 Department of Engineering Physics, Tsinghua University, 100084 Beijing, China \\ 3 Research Center of Laser Fusion, China Academy of Engineering Physics, Mianyang 621900, China \\ 4 LPCNO, Université de Toulouse, INSA, UPS, CNRS, 135 avenue de Rangueil, F-3107r Toulouse, France
}

$\begin{array}{ll}\text { PACS } & \text { 81.07.Bc - Nanocrystalline materials } \\ \text { PACS } & \text { 75.75.-c - Magnetic properties of nanostructures } \\ \text { PACS } & 68.35 .-p-\text { Solid surfaces and solid-solid interfaces: structure and energetics }\end{array}$

\begin{abstract}
Whereas bulk equiatomic FeRh alloy with B2 structure is antiferromagnetic (AFM) below $370 \mathrm{~K}$, we demonstrate that surface configuration can stabilize the low-temperature ferromagnetic (FM) state in FeRh nanoparticles in the 6-10 $\mathrm{nm}$ range. The most stable configuration for FM nanoparticles, predicted through first principles calculations, is obtained in magnetron sputtering synthesized nanoparticles. The structure, morphology and Rh-(100) surface termination are confirmed by aberration-corrected (scanning) transmission electron microscopy. The FM magnetic state is verified by vibrating sample magnetometry experiments. This combined theoretical and experimental study emphasizes the strong interplay between surface configuration, morphology and magnetic state in magnetic nanoparticles.
\end{abstract}

Close to the equiatomic composition, the chemically ordered FeRh alloy with B2 cubic structure (B2-FeRh), presents a remarkable magnetic phase transition from an antiferromagnetic (AFM) state at low temperature to a ferromagnetic (FM) state above $370 \mathrm{~K}$ [1-5]. In recent years, there has been a strong interest for this alloy for applications in microelectronics $[6,7]$, heat-assisted magnetic recording [8-10] or magnetic random access memories. [11] While the FM-AFM transition is sharp in the bulk alloy, the persistence of a FM component at low temperature in FeRh thin films raised important questions about the effect of size reduction $[12,13]$, interfaces [14-17] and surface termination $[18,19]$ on their magnetic properties. Incomplete transitions upon cooling were also reported in $\mathrm{FeRh}$ nanoparticles [20-22]. However, whereas pure size effects were studied in nanoparticles (NPs), [23] the role of surface termination remains to be addressed.

In contrast with thin epitaxial films, NPs expose different types of surfaces. In principle, their equilibrium morphology can be predicted from the hierarchy of the facet energies. [24] In chemically ordered NPs, the energy of a facet

(a)E-mail : casanove@cemes.fr can also depend on its chemical termination. Moreover, in the case of FeRh NPs, both AFM and FM states should be considered for the volume in combination with different magnetic configurations at the surface.

In this letter, we demonstrate the complex interplay between magnetic state, surface-termination and morphology in B2-FeRh nanoparticles, through a combined theoretical and experimental study. First, we present the density-functional-theory (DFT) calculations of the surface energies of AFM and FM FeRh films, for different surface magnetic configurations and terminations. These calculations reveal that Rh-terminated (100) and (111) monometallic surfaces usually have lower energies than the Fe-terminated ones, for both FM and AFM films. Remarkably, the Rh-terminated (100) surface has lower energy than the (110) surface in FM films, whereas in AFM films, the priviledged surface is the bimetallic (110) (as in body-centered cubic systems). These findings suggest a magnetic dependency of the FeRh NPs morphology. To go further, FeRh NPs, in the 6-10 nm range, were grown in a UHV device. Atomically resolved (scanning) transmission electron microscopy experiments (TEM and STEM) reveal their faceted morphology with mainly (100) and 
(110) facets and provide the first experimental evidence of the preferred Rh-termination of the (100) facets. Magnetometry measurements demonstrate the FM properties of these NPs down to 5K. Consistently, the grown FM NPs present the morphology predicted by the DFT calculations, i.e. larger Rh-terminated (100) facets compared to (110) facets, emphasizing the close relation between surface termination and magnetism.

Spin-polarized DFT calculations were performed using the VASP package [25-27] in periodic boundary conditions to study the (100), (111) and (110) surfaces. Projected augmented wave (PAW) pseudopotentials [28] were used together with the Perdew-Burke-Ernzerhoff (GGA-PBE) exchange-correlation functional $[29-31]$ and a plane wave kinetic energy cutoff of $550 \mathrm{eV}$ was employed. These calculations give the following values for bulk AFM (FM in parenthesis) B2-FeRh : lattice parameter 2.996 (3.012) $\stackrel{\circ}{\text {, }}$ Fe magnetic moment 3.14 (3.19) $\mu_{B}$, Rh magnetic moment $0.0(1.04) \mu_{B}$. The cohesive energy per unit cell is $40 \mathrm{meV}$ lower in the AFM state than in the FM state. These values compare well with experimental results [4] and previously published calculations $[5,32-34]$ performed with different exchange-correlation functionals. Three symmetric slabs, respectively ended by (100), (111) and (110) surfaces, were used. In order to use a same slab for the different magnetic states that must be considered for both the bulk (AFM or FM) and the surface layers, the lateral dimensions of the three slabs were those of the AFM unit cell, as described by Moruzzi and Marcus [5] : a square surface cell $a_{0} \sqrt{2} \times a_{0} \sqrt{2}$ for the (100) slab where $a_{0}$ is the B2 cubic cell parameter; a hexagonal cell with $a=b=a_{0} \sqrt{2}$ and $(\hat{a, b})=120^{\circ}$ for the (111) slab and a rectangular surface cell $a_{0} \sqrt{2} \times 2 a_{0}$ for the (110) slab. The different slabs with proportionally sized k-points grids, $15 \times 15 \times 1$ for (100), $13 \times 13 \times 1$ for $(111)$ and $9 \times 12 \times 1$ for $(110)$, respectively contain 15, 19 and 15 layers, and thus 30, 19 and 60 atoms. The five central atomic layers were kept fixed while the other layers were allowed to relax. To avoid the interaction between periodic slabs in the surface normal direction, the size of the vacuum region was set at $12 \AA$. Whatever the surface orientation, two different magnetic states, the AFM and FM states, were considered for the bulk. Different magnetic initial states of the topmost bilayer (surface magnetic configurations) were investigated. By convention, FM $\uparrow$ refers to the state of the FM bulk and the magnetic state at the surface is defined with reference to the magnetic state of the bulk alloy underneath. For the (100) surface, five different magnetic configurations were calculated for each surface termination ( $\mathrm{Rh}$ or $\mathrm{Fe}$ ), two for the AFM bulk (AFM and FM surface bilayers) and three for the FM $\uparrow$ bulk (AFM, FM $\uparrow$ and FM $\downarrow$ surface bilayers). A scheme of these different configurations is displayed in Figure 1. For the bimetallic (110) surface, we investigated the same five magnetic configurations as for the Rh- (or Fe) terminated (100) surface. For the (111) surface, four different magnetic configurations were calculated for each
Table 1: Calculated $\mathrm{T}=0 \mathrm{~K}$ surface energies $\left(\mathrm{J} . \mathrm{m}^{-2}\right)$ in equiatomic FeRh as a function of the magnetic surface configurations for the two different bulk magnetic orders AFM and FM

\begin{tabular}{lccccc} 
& \multicolumn{2}{c}{$\mathbf{A F M}_{\text {bulk }}$} & \multicolumn{3}{c}{$\mathbf{F M}_{\text {bulk }}$} \\
\hline \hline Surface & FM & AFM & FM $\uparrow$ & FM $\downarrow$ & AFM \\
\hline$(100)_{R h}$ & 2.174 & 2.274 & 1.775 & 1.980 & 2.034 \\
$(100)_{F e}$ & 2.649 & 2.567 & 2.559 & 2.628 & 2.646 \\
\hline \hline Surface & FM & AFM & FM $\uparrow$ & FM $\downarrow$ & \\
\hline$(111)_{R h}$ & 2.374 & 2.431 & 2.082 & 2.252 & \\
$(111)_{F e}$ & 2.366 & 2.491 & 2.181 & 2.273 & \\
\hline \hline Surface & FM & AFM & FM $\uparrow$ & FM $\downarrow$ & AFM \\
\hline$(110)$ & 2.095 & 2.090 & 1.876 & 2.147 & 2.038
\end{tabular}

surface termination ( $\mathrm{Rh}$ or $\mathrm{Fe}$ ): two for the AFM bulk (the AFM and the FM surface bilayers in which the $\mathrm{Fe}$ plane is either antiparallel (AFM) or parallel (FM) to the topmost Fe plane in the bulk) and two for FM bulk (FM $\uparrow$ and FM $\downarrow$ surface to mention here also the parallel or antiparallel alignment of the two most external Fe planes, one belonging to the surface bilayer and one to the bulk). The relaxation of the initial structures does not change the surface and bulk magnetic states but slightly modifies the magnetic moments of $\mathrm{Fe}$ and $\mathrm{Rh}$ atoms located at the surface or in the topmost bulk layers.

The surface energy per unit area for a symmetric slab is simply defined as half the energy necessary to break an infinite crystal into two pieces:

$$
\gamma=\frac{1}{2 S}\left(E_{s l a b}-E_{b u l k}\right)
$$

where S denotes the surface area at equilibrium. As the slabs expose the same top and bottom surfaces, they are off-stoichiometry in the case of (100) and (111) films. As a consequence, the bulk energy $E_{\text {bulk }}$ must be calculated in each different slab using :

$$
E_{\text {bulk }}=N_{F e} \mu_{F e}+N_{R h} \mu_{R h}
$$

where $N_{F e}$ and $N_{R h}$ are the numbers of Fe and Rh atoms in the alloy and $\mu_{F e}$ and $\mu_{R h}$ their chemical potentials at $0 \mathrm{~K}$. The chemical potentials have been calculated in both AFM and FM states following the combined $a b$ initio and statistical mechanics approach ${ }^{1}$ described in [35-37].

The computed surfaces energies as a function of the magnetic configurations are gathered in Table 1. Independently of the bulk magnetic state, the Rhterminated FM surface configuration is much more

\footnotetext{
${ }^{1}$ The statistical model uses as input data the grand canonical DFT formation energies of the four elementary point defects, i.e. vacancy and antisite defects on both Fe and Rh sublattices. These formation energies have been calculated with large simulation periodic supercells containing the considered defect.
} 

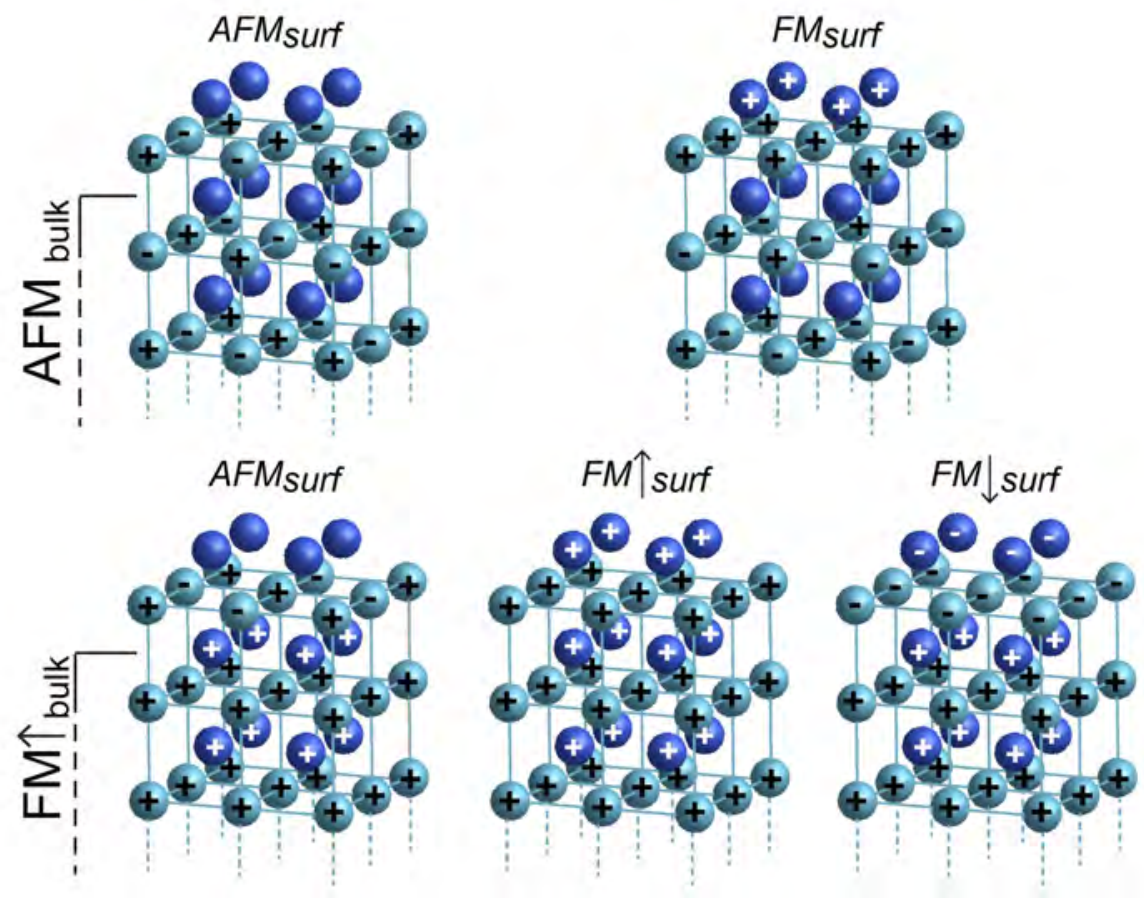

Fig. 1: Scheme of the five magnetic surface configurations considered for the calculation of the Rh-terminated (100) surface: two for AFM bulk (top) and three for FM bulk (bottom). The + and - signs in the atoms (Fe:light blue; Rh: dark blue) indicate parallel or antiparallel coupling of the magnetic moments.

favorable for the (100) surface. This result is consistent with both theoretical predictions [18] on ultrathin free-standing films and recently reported experimental studies of the surface of a Rh-terminated (100) FeRh films. [38] For (111) surfaces, our calculations show mixed results but the Rh-termination remains slightly more favorable in most cases. Most interestingly, comparing several low-index surfaces allows us to reveal a magnetic order dependency of the hierarchy of the surface energies. Indeed, in films with AFM bulk, the surface with lower energy is the dense (110) bimetallic surface, whatever the magnetic configuration of the surface and its chemical termination. This is not the case for the FM bulk state as the Rh-terminated (100) surface becomes more favorable than the (110) surface in all the different magnetic surface configurations. Experiments have been conducted in order to investigate the interplay between facet competition and magnetic properties in nanoparticles.

Fe-Rh NPs were grown by dc magnetron co-sputtering from two elemental targets in a ultra-high vacuum (UHV) chamber. The growth temperature was set to $730{ }^{\circ} \mathrm{C}$. A 5 $\mathrm{nm}$ thick amorphous alumina layer $\left(a-\mathrm{Al}_{2} \mathrm{O}_{3}\right)$ was used as a substrate (itself deposited on a thin $\mathrm{NaCl}$ crystal). After metal deposition, the temperature was slowly decreased to room temperature and maintained at this temperature to deposit a $5 \mathrm{~nm}$ thick $a-\mathrm{Al}_{2} \mathrm{O}_{3}$ cover layer in order to prevent oxidation and NPs coalescence. [39]
The FeRh NPs embedded in the alumina matrix were transferred on copper grids for TEM-STEM investigations, after dissolution of the $\mathrm{NaCl}$ support. Their analyses were conducted using Cs-corrected instruments (for objective lens or probe), a FEI Tecnai F20 and a Jeol cold FEG ARM200F microscopes. Energy x-ray dispersive spectroscopy (EDS) performed on a large assembly of nanoparticles revealed a mean composition of $\mathrm{Fe}_{47} \mathrm{Rh}_{53}$, which agrees with the composition range for AFM B2FeRh, $0.44<x_{F e}<0.52$. [40]

Figure 2 summarizes the findings of the TEM analysis. The low magnification image and associated size histogram show that the NPs have a narrow size dispersity (standard deviation of $1.5 \mathrm{~nm}$ ) with a mean diameter of 8.4 $\mathrm{nm}$. The NPs appear fully cristalline as the one displayed in the inset in figure 2-a and display neat (100) and (110) facets when observed along a [001] direction. The numerical diffractogram (figure 2-c) recorded from the Fourier transform of a high resolution image of an assembly of these NPs (not shown) displays a set of reflexions fully consistent with B2-FeRh, in particular a strong 100 reflexion (forbidden in bcc). Contrary to room-temperature grown NPs (see for instance $[20,22,23]$ ), no annealing step is thus required to achieve the B2 order in the present case, the growth temperature being high enough to favor atomic diffusion and chemical order.

The chemical nature of the atomic columns in the FeRh NPs is analyzed in HAADF-STEM mode. Thanks to the 


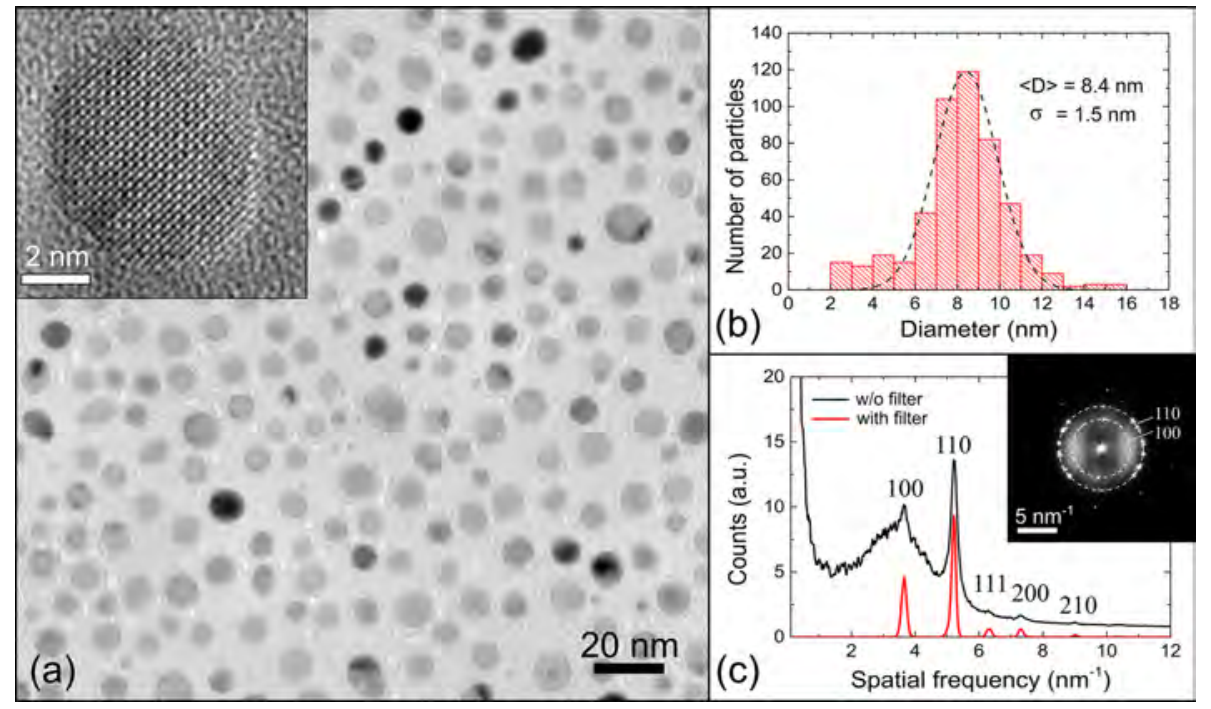

Fig. 2: a) TEM view of the grown Fe-Rh NPs together with a HREM image of a NP in the inset ; b) Corresponding histogram of size distribution fitted with a gaussian distribution; c) Intensity profile of the numerical diffraction pattern (shown in the inset), displayed with and without the amorphous background due to alumina.

Z-dependency of the contrast, the Rh atomic columns appear much brighter than the Fe ones in Figure 3. Our analysis of the exposed facets on suitably oriented NPs provides the first experimental evidence for the Rhtermination of the (100) facets in FeRh NPs. This preferred termination is clearly observed in profile 3 of Figure 3. In this orientation, the NPs present large (100) facets and small (110) bimetallic facets. According to the Wulff theorem, $\frac{\gamma_{i}}{h_{i}}=$ constant, where $\gamma_{i}$ refers to the surface energy per unit area of the $i$ facet and $h_{i}$ refers to the distance of the $i$-facet to the nanoparticle center. The ratio $h_{100}^{e x p} / h_{110}^{e x p}$ of the distances from the center of the nanoparticle to the two different facets thus provides a rather good estimate of the ratio of their surface energies: $\gamma_{100}^{\exp } / \gamma_{110}^{\exp } \simeq 0.91$. This value is very close to the ratio of the calculated surface energies corresponding to the more stable (i.e. $F M \uparrow$ ) surface configuration in the $F M \uparrow_{\text {bulk }}$ case (see Table 1), with Rh-terminated (100) surface: $\gamma_{100}^{\text {calc }} / \gamma_{110}^{\text {calc }}=h_{100}^{\text {calc }} / h_{110}^{\text {calc }}=0.946$. Note that all the investigated NPs present a $h_{100}^{\exp } / h_{110}^{\exp }$ ratio lower than 1. The inset in Figure 3 displays the Wulff polyhedron built using these calculated values. In contrast, the observed morphology poorly agrees with the one that can be derived from the surface energies hierarchy in the most stable case of the Rh-terminated $A F M_{b u l k}$ configuration (first column in Table 1) with $\gamma_{100}^{\text {calc }} / \gamma_{110}^{\text {calc }}$ greater than 1 . As our NPs present a slight Fe under-stoichiometry, we also calculated the surface energies for a $4 \%$ deviation from stoichiometry by replacing in equation (2), the $\mathrm{T}=0 \mathrm{~K}$ chemical potentials of the equiatomic alloy by the ones calculated for the $\mathrm{Fe}_{0.48} \mathrm{Rh}_{0.52}$ alloy. Table 2 presents the corresponding surface energies for the FM bulk state. The $F M \uparrow$ with Rh-termination remains the more favorable surface configuration and the $\gamma$ ratio is now closer to the one deduced from experiments $\gamma_{100}^{\text {calc }} / \gamma_{110}^{\text {calc }}=0.89$. Moreover, assuming that the main contribution to the difference of surface free energy between the two surfaces comes from the chemical potentials, the $\gamma$ ratio calculated with the $\mathrm{T}$ $=300 \mathrm{~K}$ chemical potentials reaches 0.91 , i.e. the experimental value. The facet competition thus strongly privileges the Rh-terminated (100) facets over other facets in FM bulk and the effect is reinforced by a slight Rh enrichment. The reported morphology thus strongly suggests the presence of the FM state in the analyzed NPs.

The magnetic properties of an assembly of these NPs were measured using vibrating sample magnetometry (VSM) in a PPMS Quantum Design system. Magnetization data were recorded along the sample plane. Figure 4-a presents magnetization measurements according to a zero-field cooling (ZFC)-field cooling (FC) procedure for different applied magnetic fields. They give evidence for a superparamagnetic behavior of the assembly with a blocking temperature about $150 \mathrm{~K}$. The blocking temperature decreases when increasing the magnetic field, which is characteristic for a superparamagnetic nanomaterial. Some representative isothermal $M(H)$ hysteresis loops measured at different temperatures from $390 \mathrm{~K}$ to $5 \mathrm{~K}$ are presented in Figure 4-b. All these measurements were performed after a FC from $390 \mathrm{~K}$ down to $2.5 \mathrm{~K}$ under a field of $6 \mathrm{~T}$. None of the $M(H)$ displayed an exchange bias phenomena that could be expected for a ferromagnetic / antiferromagnetic interface. The evolution of the coercive field $H_{c}$ with temperature is plotted in the inset. It confirms the superparamagnetic transition above $150 \mathrm{~K}$, as $H_{c}$ goes to zero. The saturation magnetization $\left(M_{S}\right)$ is obtained in moderate magnetic fields, below $1 \mathrm{~T}$, and 


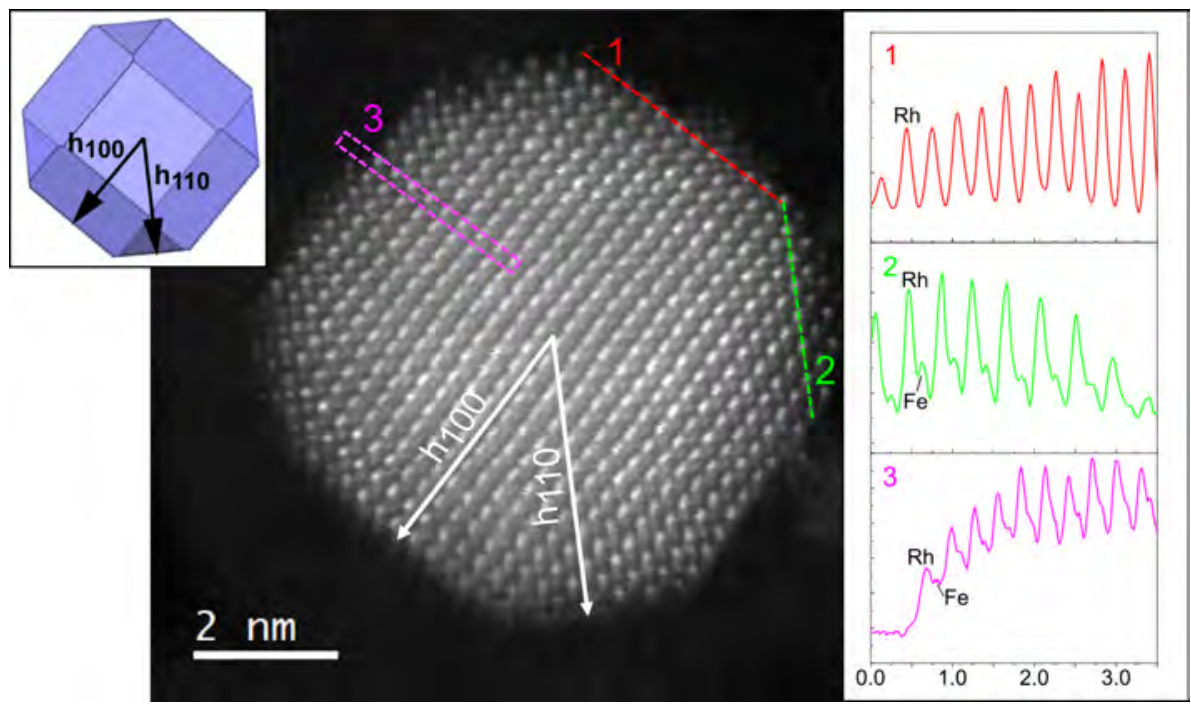

Fig. 3: HAADF-STEM image of a NP observed along a [001] direction of B2-FeRh. Intensity profiles along different lines are presented on the right. Profiles 1 and 2 respectively correspond to a [100] raw of Rh atomic columns and a [110] raw showing alternate $\mathrm{Fe}$ and $\mathrm{Rh}$ columns. Profile 3 displays the projected intensities of two adjacent raws (Fe and Rh raws) along a [100] direction drawn from the surface of the NP to its center. The scheme in inset corresponds to the calculated Wulff polyhedron.

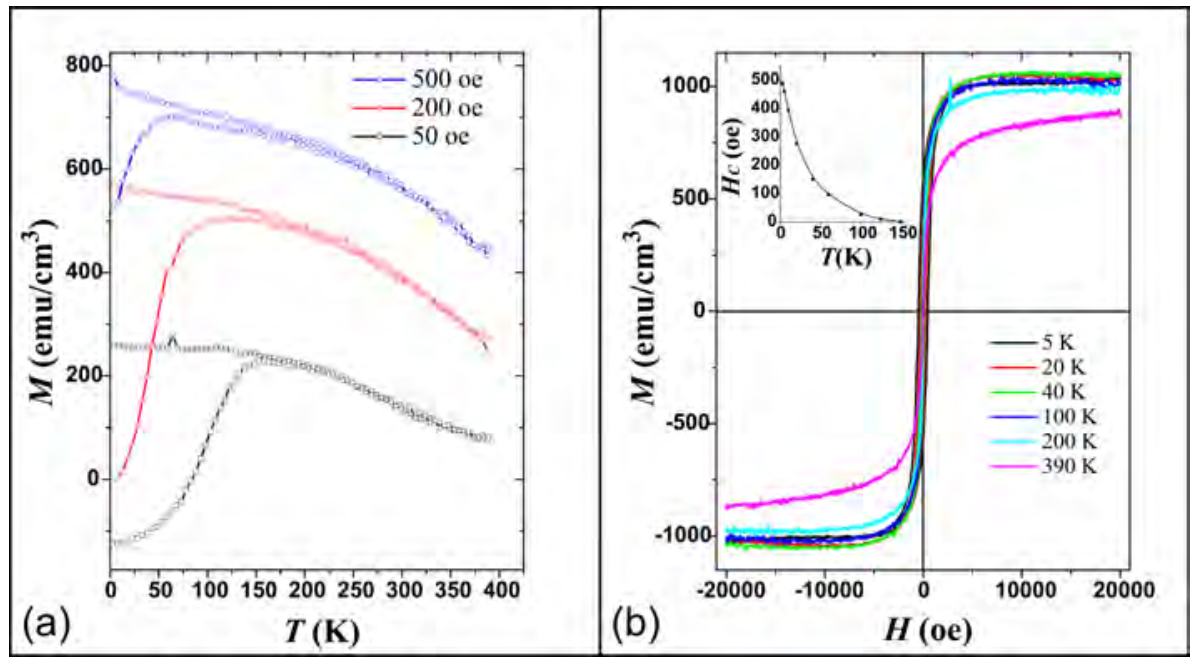

Fig. 4: a) ZFC/FC magnetization measurements of the FeRh NPs for different applied fields ; b) Isothermal $M(H)$ hysteresis loops recorded from $5 \mathrm{~K}$ to $390 \mathrm{~K}$ after a $\mathrm{FC}$ under $6 \mathrm{~T}$ from $390 \mathrm{~K}$. The inset shows the evolution of the coercive field $H_{c}$ with temperature. 
Table 2: Calculated $\mathrm{T}=0 \mathrm{~K}$ surface energies $\left(J . \mathrm{m}^{-2}\right)$ in $F M_{\text {bulk }} \mathrm{Fe}_{0.48} \mathrm{Rh}_{0.52}$.

\begin{tabular}{lccc} 
Surface & $F M \uparrow$ & $F M \downarrow$ & $A F M$ \\
\hline \hline$(100)_{R h}$ & 1.677 & 1.882 & 1.936 \\
\hline$(100)_{F e}$ & 2.657 & 2.725 & 2.744 \\
\hline \hline$(110)$ & 1.876 & 2.147 & 2.038
\end{tabular}

slowly and monotonically decreases upon increasing the temperature. This set of data is a clear indication of the FM state in the NPs, even at very low temperature. According to the experimental values (see [4] for instance), the magnetization reaches around $4 \mu_{B}$ per FeRh unit cell in the FM state and thus around $1370 \mathrm{emu} / \mathrm{cm}^{3}$. This value is however usually not reached in thin films. Our estimate of $M_{S},\left(1050( \pm 200) \mathrm{emu} / \mathrm{cm}^{3}\right)$ is in reasonable agreement with the magnetization in the bulk alloy even if more precise measurements are foreseen to reduce the uncertainty of our estimate. These results confirm without any ambiguity the persistence of the FM state down to $5 \mathrm{~K}$ in equiatomic $\mathrm{B} 2-\mathrm{FeRh}$ nanoparticles in the $6-10$ $\mathrm{nm}$ range, without any evidence for an even partial FM to AFM transition upon cooling in the range of field (up to 8T) and temperature experienced.

In summary, using DFT calculations, we compared the energies of low-index surfaces in different magnetic configurations for both the surface and the bulk in equiatomic FeRh. While confirming the enhanced stability of Rhterminated FM surface configuration for the (100) surface, as reported for thin FeRh films, our results strongly emphasize the influence of the bulk magnetic order on the exposed surface hierarchy. In particular, the mentioned facet is much more stable than the other facets in case of a FM bulk state. Consistently, we provide the first evidence for the occurrence of large Rh-terminated (100) facets in grown NPs with B2 structure. Our magnetic measurements demonstrate that the synthesized NPs are ferromagnetic despite their relatively large size $(\simeq 8 \mathrm{~nm})$. There is presently an agreement on the role played by the Rh magnetic moment, induced by a spin-dependent hybridization with surrounding Fe atoms, in the stabilization of the FM state in the bulk alloy above a given temperature $[33,34]$. The stabilization of the FM state in our nanoparticles can be accounted for the enhanced polarization of $\mathrm{Rh}$ at the large Rh-terminated (100) surface facets, as observed in thin films [18,38], which favors the formation of a FM shell. Clearly, the magnetic state at the surface impedes the stabilization of the AFM state in the nanocrystal core, at this size range. Our results thus strongly suggest the important role played by the surface configuration in the morphology of FeRh NPs and in the stabilization of the FM state. It also opens perspectives for tuning the finite-size properties of this remarkable alloy and suggests new strategies for designing AFM-core-FM shell nanoparticles through size and morphology control.
This work was supported by the French Ministry of Foreign and European Affairs (M.L. PhD grant). It was performed using HPC resources from CALMIP (Grant 2015P0933). We gratefully acknowledge for their assistance, Lucien Datas and Teresa Hungria with the ARM200F at Centre de Microcaracterization R. Castaing and Sebastien Pinaud for the magnetization measurements at LPCNO.

\section{REFERENCES}

[1] Fallot M., Annales de Physique (Paris), 10 (1938) 291.

[2] Fallot M. and Hocart R., Rev. Sci., 77 (1939) 498.

[3] Kouvel J. S. and Hartelius C. C., Journal of Applied Physics, 33 (1962) 1343.

[4] Shirane G., Nathans R. and Chen C. W., Phys. Rev., 134 (1964) A1547.

[5] Moruzzi V. L. and Marcus P. M., Phys. Rev. B, 46 (1992) 2864.

[6] Ju G., Hohlfeld J., Bergman B., van de VeerDONK R. J. M., Mryasov O. N., Kim J.-Y., WU X., Weller D. and Koopmans B., Phys. Rev. Lett., 93 (2004) 197403.

[7] Radu I., Stamm C., Pontius N., Kachel T., Ramm P., Thiele J.-U., Dürr H. A. and Back C. H., Phys. Rev. B, 81 (2010) 104415.

[8] Thiele J.-U., Maat S. and Fullerton E. E., Applied Physics Letters, 82 (2003) 2859.

[9] Thiele J.-U., Maat S., Robertson J. and Fullerton E., Magnetics, IEEE Transactions on, 40 (2004) 2537.

[10] Cherifi R. O., Ivanovskaya V., Phillips L. C., ZoBelli A., Infante I. C., Jacquet E., Garcia V., Fusil S., Briddon P. R., Guiblin N., Mougin A., nal A. A., Kronast F., Valencia S., Dkhil B., Barthlmy A. and Bibes M., Nat Mater, 13 (2014) 345.

[11] Marti X., Fina I., Frontera C., Liu J., Wadley P., He Q., Paull R. J., Clarkson J. D., Kudrnovsk J., Turek I., Kune J., Yi D., Chu J.-H., Nelson C. T., You L., Arenholz E., Salahuddin S., Fontcuberta J., Jungwirth T. and Ramesh R., Nat Mater, 13 (2014) 367.

[12] Suzuki I., Koike T., Iтoh M., Taniyama T. and Sato T., Journal of Applied Physics, 105 (2009) $07 \mathrm{E} 501$.

[13] Han G. C., Qiu J. J., Yap Q. J., Luo P., Laughlin D. E., Zhu J. G., Kanbe T. and Shige T., Journal of Applied Physics, 113 (2013)

[14] Fan R., Kinane C. J., Charlton T. R., Dorner R., Ali M., de Vries M. A., Brydson R. M. D., Marrows C. H., Hickey B. J., Arena D. A., Tanner B. K., Nisbet G. and Langridge S., Phys. Rev. B, 82 (2010) 184418.

[15] Baldasseroni C., Bordel C., Gray A. X., Kaiser A. M., Kronast F., Herrero-Albillos J., Schneider C. M., Fadley C. S. and Hellman F., Applied Physics Letters, 100 (2012).

[16] Bordel C., Juraszek J., Cooke D. W., BaLDasseroni C., Mankovsky S., Minár J., Ebert H., Moyerman S., Fullerton E. E. and Hellman F., Phys. Rev. Lett., 109 (2012) 117201. 
[17] Baldasseroni C., Plsson G. K., Bordel C., Valencia S., Unal A. A., Kronast F., Nemsak S., Fadley C. S., Borchers J. A., Maranville B. B. and HellMAN F., Journal of Applied Physics, 115 (2014) .

[18] Lounis S., Benakki M. and Demangeat C., Phys. Rev. $B, 67$ (2003) 094432.

[19] Jeknl S., Rhim S. H., Hong S. C., Son W.-J. and Shick A. B., Phys. Rev. B, 92 (2015) 064410.

[20] Jia Z., Harrell J. W. and Misra R. D. K., Applied Physics Letters, 93 (2008) 022504.

[21] Ko H. Y. Y. and Suzuki T., Magnetics, IEEE Transactions on, 43 (2007) 885 .

[22] Ko H. Y. Y., Suzuki T., Phuoc N. N. and CaO J., Journal of Applied Physics, 103 (2008) 07D508.

[23] Hillion A., Cavallin A., Vlaic S., Tamion A., Tournus F., Khadra G., Dreiser J., Piamonteze C., Nolting F., Rusponi S., Sato K., Konno T. J., Proux O., Dupuis V. and Brune H., Phys. Rev. Lett., 110 (2013) 087207.

[24] WulfF G., Zeitschrift fur Krystallographie und Mineralogie, 34 (1901) 449.

[25] Kresse G. and Hafner J., Phys. Rev. B, 47 (1993) 558.

[26] Kresse G. and Furthmüller J., Phys. Rev. B, 54 (1996) 11169.

[27] Kresse G. and Furthmüller J., Comput. Mater. Sci., 6 (1996) 15.

[28] Blöchl P. E., Phys. Rev. B, 50 (1994) 17953.

[29] Perdew J. P., Burke K. and Ernzerhof M., Phys. Rev. Lett., 77 (1996) 3865.

[30] Langreth D. C. and Perdew J. P., Phys. Rev. B, 21 (1980) 5469.

[31] Langreth D. C. and Mehl M. J., Phys. Rev. Lett., 47 (1981) 446.

[32] Deák A., Simon E., Balogh L., Szunyogh L., dos Santos Dias M. and Staunton J. B., Phys. Rev. B, 89 (2014) 224401.

[33] Kudrnovský J., Drchal V. and Turek I., Phys. Rev. $B, 91$ (2015) 014435.

[34] Polesya S., Mankovsky S., Ködderitzsch D., Minár J. and Ebert H., Phys. Rev. B, 93 (2016) 024423.

[35] Mayer J., Elsässer C. and Fähnle M., Physica Status Solidi (b), 191 (1995) 283.

[36] Meyer B. and Fähnle M., Phys. Rev. B, 59 (1999) 6072.

[37] Besson R., Legris A. and Morillo J., Phys. Rev. B, 74 (2006) 094103.

[38] Pressacco F., Uhl V., Gatti M., Bendounan A., Fullerton E. E. and Sirotti F., Scientific Reports, 6 (2016) 22383.

[39] Langlois C., Benzo P., Arenal R., Benoit M., Nicolai J., Combe N., Ponchet A. and Casanove M. J., Nano Letters, 15 (2015) 5075 pMID: 26146846.

[40] Swartzendruber L., Bulletin of Alloy Phase Diagrams, 5 (1984) 456. 